\title{
An upper limit on the cosmic-ray luminosity of individual sources from gamma-ray observations
}

\author{
A.D. Supanitsky, ${ }^{a}$ V. de Souza ${ }^{b}$ \\ ${ }^{a}$ Instituto de Astronomía y Física del Espacio (IAFE), CONICET-UBA, Argentina \\ ${ }^{b}$ Instituto de Física de São Carlos, Universidade de São Paulo, Brazil \\ E-mail: supanitsky@iafe.uba.ar, vitor@ifsc.usp.br
}

\begin{abstract}
Different types of extragalactic objects are known to produce $\mathrm{TeV}$ gamma-rays. Some of these objects are the most probable candidates to accelerate cosmic rays up to $10^{20}$ $\mathrm{eV}$. It is very well known that gamma-rays can be produced as a result of the cosmic ray propagation through the intergalactic medium. These gamma-rays contribute to the total flux observed in the direction of the source. In this paper we propose a new method to derive an upper limit on the cosmic-ray luminosity of an individual source based on the measured upper limit on the integral flux of $\mathrm{GeV}-\mathrm{TeV}$ gamma-rays. We show how it is possible to calculate an upper limit on the cosmic-ray luminosity of a particular source and we explore the parameter space in which the current $\mathrm{GeV}-\mathrm{TeV}$ gamma-ray measurements can offer a useful determination. We study in detail two particular sources, Pictor A and NGC 7469, and we calculate the upper limit on the proton luminosity of each source based on the upper limit on the integral gamma-ray flux measured by the H.E.S.S. telescopes.
\end{abstract}




\section{Contents}

1 Introduction 1

2 The method 3

3 Application of the method 4

3.1 Pictor A 6

$\begin{array}{lll}3.2 & \text { NGC } 7469 & 7\end{array}$

4 Conclusions $\quad 8$

$\begin{array}{ll}\text { A Simulations } & 8\end{array}$

B Contribution of a point source to the measured UHECR flux 9

\section{Introduction}

Individual sources of ultra high energy cosmic rays (UHECR) ${ }^{1}$ with energy above $10^{18} \mathrm{eV}$ have not been identified yet due to the severe limitations imposed by the uncertainties on the determination of the identity of the particles and the unknown strength and structure of the magnetic fields in the Universe. The reconstructed direction of a charged cosmic-ray particle might not point back exactly to its source due to deviation in the magnetic fields. Given this limitation, the study of the origin of the cosmic rays is restricted to limited classes of sources and any important information involves the convolution of measured quantities with propagation models and catalogs of objects [1].

One possible exception to this scenario which needs extra data to be confirmed is Cen A. The Pierre Auger Observatory has measured an excess of events around Cen A [1], however the correlation angle is very large $(\sim 18$ degrees $)$ and many other objects lie within this angular window.

On the other hand, gamma-rays are not deviated by magnetic fields. Cosmic-ray observatories are sensitive to gamma-rays at the highest energies $\left(E_{\gamma} \gtrsim 10^{18} \mathrm{eV}\right)$. At present, no observation of a high energy photon has been claimed, all candidate events are compatible with nuclei primaries. As a consequence upper limits on the gamma-ray flux at the highest energies have been obtained [2]. At TeV energies a few hundred gamma-ray sources have been discovered by the past and current Imaging Atmospheric Cherenkov Telescopes (IACTs). Also, at lower energies $(\mathrm{GeV})$ more than one thousand gamma-ray sources have been detected by the Fermi-LAT instrument [3]. It is believed that the majority of the gamma-rays observed in the $\mathrm{GeV}-\mathrm{TeV}$ energy range originate in those sources. However a significant number of these gamma-rays can be produced in the propagation of cosmic rays in the intergalactic medium [4-8].

UHECRs interact with the radiation backgrounds producing secondary ${ }^{2}$ particles. The production of secondary particles in the cosmic microwave background and in the extragalactic

\footnotetext{
${ }^{1}$ Hereafter cosmic rays will refer to the hadronic component of the total flux. Photons and neutrinos can be part of the flux observed at Earth but they are a minority component.

${ }^{2}$ The name primary is used for particles (cosmic rays and gamma-rays) produced in the sources. Here, the word secondary names particles produced on the way from the source to Earth.
} 
background light has been studied in detail (see for instance [9-11]). The relevant aspect of the propagation of UHECR for this paper is the production of $\mathrm{GeV}-\mathrm{TeV}$ gamma-rays which can be summarized as follows.

Pions, electron-positron pairs, and neutrons are the main outcome of the interaction of the primary cosmic ray with the radiation fields. For the considered distances (extragalactic sources) pions and neutrons decay before reaching Earth. Gammas, electrons and neutrinos are the most numerous secondary particles produced in the cascade process. Gamma-rays $(E \gtrsim 10 \mathrm{GeV})$ interact with the low energy photons of the radiation fields producing electronpositron pairs. Electrons and positrons can upscatter low energy photons of the background via inverse Compton producing gamma-rays that continue the cascade process.

The relevant photon backgrounds for this cascading process are: (a) The extragalactic background light composed of the infrared, visible, and ultraviolet regions, which is important in the energy range from $E_{\gamma}=10 \mathrm{GeV}$ to $E_{\gamma}=10^{5} \mathrm{GeV}$ of the incident gamma, (b) the cosmic microwave background from $E_{\gamma}=10^{5} \mathrm{GeV}$ to $E_{\gamma}=10^{10} \mathrm{GeV}$, and (c) the radio background from $E_{\gamma}=10^{10} \mathrm{GeV}$ to $E_{\gamma}=10^{13} \mathrm{GeV}$ [12].

The cascade process generates a high flux of secondary photons with energy in the GeV$\mathrm{TeV}$ range. Due to the production of secondary gamma-rays in the propagation of cosmic rays in the intergalactic medium, the number of gamma-rays measured at any given direction in the sky is a combination of the ones produced in the source (primary flux) and the ones produced during the propagation of the cosmic rays (secondary flux). This is an ambiguity which has to be solved in order to extract the primary gamma-ray spectrum of a source. In fact, it has been pointed out that the measured gamma-ray spectra of some unusually bright distant blazars might originate as a result of the propagation of high energy protons emitted by these sources [13-15].

Among all candidate sources observed by the ground and space based observatories, there are some for which only an upper limit on the gamma-ray flux is obtained. For these objects the upper limit constrains the sum of the primary and secondary fluxes of photons. If the measured upper limit flux of $\mathrm{GeV}-\mathrm{TeV}$ gamma-rays is interpreted as the upper limit on the secondary gamma-rays produced by the cosmic rays propagating from a distance source to Earth, it is possible to set an upper limit on the cosmic-ray luminosity of the source. If the primary gamma-ray flux of the source is not taken into account then the calculated upper limit on the cosmic-ray luminosity is the most conservative.

The method proposed here relies on calculations of the UHECR propagation in the intergalactic medium to establish the connection between the UHECR and GeV-TeV gammarays fluxes. The method can be applied to any upper limit on the $\mathrm{GeV}-\mathrm{TeV}$ gamma-ray flux measured by ground or space based instruments. In order to illustrate the method we have used a catalog of sources for which the H.E.S.S. telescopes have measured upper limits [16].

Two particular sources Pictor A and NGC 7469 are studied in detail. Pictor A is a Fanarof-Riley II galaxy with high radio activity. Its jet is clearly visible by Chandra [17] and it is oriented at a large angle with respect to Earth, therefore the gamma-ray emission is not expected to be high. The H.E.S.S. telescopes observed Pictor A for 7.9 h, no signal was detected and an upper limit on the integral flux of gamma-rays was obtained. NGC 7469 is a Seyfert 1 spiral galaxy placed at $\sim 68 \mathrm{Mpc}$ from the Earth. It has been observed by H.E.S.S. for $3.4 \mathrm{~h}$, no excess was seen and an upper limit on the integral flux of gamma-ray was also calculated. The method developed in this paper allows us to calculate an upper limit on the proton luminosities of Pictor A and NGC 7469, for energies above $10^{18} \mathrm{eV}$, based on the upper limits on the integral flux of gamma-ray obtained by H.E.S.S.. 
The new method proposed here has its relevance increased by the proposed construction of the CTA Observatory [18]. The CTA Observatory will have a sensitivity one order of magnitude better than the current IACTs and therefore is going to be able to set very strict upper limits at $\mathrm{GeV}-\mathrm{TeV}$ energies.

At the same time, the scientific community is planning the next steps for the detection of UHECR [19, 20]. Multi-messenger astroparticle physics has been claimed to be the key to solving the remaining puzzles in high energy astrophysics [21-23]. The idea presented in this paper contributes in this direction.

\section{The method}

The method developed in this section establishes the connection between a measured upper limit on the integral flux of GeV-TeV gamma-rays of a source and the UHECR cosmic-ray luminosity of the same source. The purpose of this section is to formalize the method in a general mathematical way. The details of the application of the method to specific sources are done in section 3 .

Let us start by assuming that a point source injects a cosmic ray energy spectrum given by a power law with an exponential cutoff

$$
\frac{d N}{d E d t}=\frac{L_{C R}}{C_{0}} E^{-\alpha} \exp \left(-E / E_{c u t}\right)
$$

where $L_{C R}$ is the cosmic-ray luminosity, $\alpha$ is the spectral index, $E_{c u t}$ is the cutoff energy, and $C_{0}$ is a normalization constant

$$
C_{0}=\int_{E_{\min }}^{\infty} d E E^{-\alpha+1} \exp \left(-E / E_{c u t}\right)
$$

where $E_{\min }=10^{18} \mathrm{eV}$ is the minimum energy of a cosmic-ray particle produced by the source.

The injection spectrum can be rewritten as

$$
\frac{d N}{d E d t}=\frac{L_{C R}}{\langle E\rangle_{0}} P_{C R}^{0}(E)
$$

where $P_{C R}^{0}(E)$ is the normalized energy distribution of the injected particles

$$
P_{c r}^{0}(E)=\frac{E^{-\alpha} \exp \left(-E / E_{c u t}\right)}{\int_{E_{\min }}^{\infty} d E E^{-\alpha} \exp \left(-E / E_{c u t}\right)}
$$

and $\langle E\rangle_{0}$ is the mean energy

$$
\langle E\rangle_{0}=\int_{E_{\min }}^{\infty} d E E P_{C R}^{0}(E)=\frac{\int_{E_{\min }}^{\infty} d E E^{-\alpha+1} \exp \left(-E / E_{c u t}\right)}{\int_{E_{\min }}^{\infty} d E E^{-\alpha} \exp \left(-E / E_{c u t}\right)} .
$$

On the way from the source to Earth, cosmic ray particles interact with radiation fields causing a modification of the energy distribution from $P_{C R}^{0}(E)$ to $P_{C R}(E)$. For a source at 
a given comoving distance $\left(D_{s}\right)$ from Earth, assuming an isotropic emission, the cosmic-ray flux can be written as

$$
I_{C R}(E)=\frac{L_{C R}}{4 \pi D_{s}^{2}\left(1+z_{s}\right)\langle E\rangle_{0}} K_{C R} P_{C R}(E),
$$

where $z_{s}$ is the redshift of the source, $P_{C R}(E)$ is the energy distribution of particles arriving on Earth and $K_{C R}$ is the number of cosmic rays arriving on Earth per injected particle. $K_{C R}$ and $P_{C R}(E)$ include the physics of the propagation of the cosmic rays, i.e. all interactions and energy losses suffered during the propagation process. Here an isotropic cosmic ray emission is assumed.

This source generates a secondary gamma-ray flux which is proportional to its cosmicray luminosity. In analogy to equation (2.6) it is possible to write the gamma-ray flux at Earth as a function of the cosmic-rays luminosity,

$$
I_{\gamma}\left(E_{\gamma}\right)=\frac{L_{C R}}{4 \pi D_{s}^{2}\left(1+z_{s}\right)\langle E\rangle_{0}} K_{\gamma} P_{\gamma}\left(E_{\gamma}\right)
$$

where $K_{\gamma}$ is the number of gamma-rays generated per injected cosmic-ray particle and $P_{\gamma}\left(E_{\gamma}\right)$ is the energy distribution of the gamma-rays arriving on Earth. Here $E_{\gamma}$ is used to denote the energy of the gamma-rays. Note that in this case $K_{\gamma}$ and $P_{\gamma}\left(E_{\gamma}\right)$ include the physics of the propagation of the cosmic-rays and gamma-rays.

Equation (2.7) relates the gamma-ray flux measured on Earth to the cosmic-ray luminosity. Therefore, an upper limit on the integral gamma-ray flux can be translated into an upper limit on the cosmic-ray luminosity $\left(L_{C R}^{U L}\right)$ of the source according to

$$
L_{C R}^{U L}=I_{\gamma}^{U L}\left(>E_{\gamma}^{t h}\right) \frac{4 \pi D_{s}^{2}\left(1+z_{s}\right)\langle E\rangle_{0}}{K_{\gamma} \int_{E_{\gamma}^{t h}}^{\infty} d E P_{\gamma}\left(E_{\gamma}\right)},
$$

where $I_{\gamma}^{U L}\left(>E_{\gamma}^{t h}\right)$ is the measured upper limit on the integral gamma-ray flux obtained above a given energy threshold $\left(E_{\gamma}^{\text {th }}\right)$ at a given confidence level (CL).

Equation (2.8) is the summary of the method presented here. It allows us to calculate an upper limit on the cosmic-ray luminosity given the measured upper limit on the integral gamma-ray flux. $K_{\gamma}$ and $P_{\gamma}\left(E_{\gamma}\right)$ summarize the propagation process under the assumption of the source properties: distance $\left(D_{S}\right)$ and injection spectrum $\left(P_{C R}^{0}(E)\right)$.

In general, the factors $K_{C R}$ and $K_{\gamma}$ and the distribution functions $P_{C R}(E)$ and $P_{\gamma}\left(E_{\gamma}\right)$ can be obtained from Monte Carlo simulations [24] (see Appendix A for details).

It is worth noting that when a mixture of nuclei are injected by the source such that the proton fraction is different from zero, equation (2.8) when calculated for proton primaries (using $P_{\gamma}^{p r}$ and $K_{\gamma}^{p r}$ ) gives an upper limit on the proton luminosity of the source. This can be understood from the fact that, if heavier nuclei are injected by the source in addition to proton primaries, the resulting gamma-ray flux at Earth is larger than the one expected for protons only.

\section{Application of the method}

The data considered in this work were taken by the H.E.S.S. telescopes [16]. H.E.S.S. is an important ground-based gamma-ray observatory currently in operation that makes use of 
the imaging atmospheric Cherenkov technique. Between 2005 and 2007, H.E.S.S. observed several active galactic nuclei from which a gamma-ray flux with energy above $100 \mathrm{GeV}$ was expected to be measured. At this energy, H.E.S.S.'s sensitivity is at the level of a few percent of the Crab Nebula flux.

In Ref. [25] the H.E.S.S. Collaboration reported the observation of 14 candidate sources for which no significant emission was detected. From the 14 candidate sources, 10 were Blazar and 4 Non-Blazar types of AGNs. In that publication the upper limit on the integral gammaray flux of each source was reported. We select three sources from the H.E.S.S. publication to illustrate the method proposed here: NGC 1068, NGC 7469, and Pictor A. These three sources were chosen by a combination of factors. They are all non-Blazar, they cover the distance range of interest $(\sim 16, \sim 68$, and $\sim 141 \mathrm{Mpc})$, and they are all in the field of view of the Pierre Auger Observatory.

The factor $K_{\gamma}$ and the energy distribution $P_{\gamma}\left(E_{\gamma}\right)$ for each of the three sources were calculated by using the Monte Carlo simulation program CRPropa [24] (see Appendix A for details) under the following input assumptions. The injection spectrum is a power law with an exponential cutoff as shown by equation (2.1). $E_{\text {cut }}$ is taken to be proportional to the charge number $(Z)$ of the injected particle $E_{c u t}^{Z}=Z \times E_{c u t}^{p r}$, where $p r=$ proton. Two cases were simulated: Only proton and only iron nuclei as primary particles. The following cutoff energies were considered: $\log \left(E_{c u t}^{p r} / \mathrm{eV}\right)=20,20.25,20.5,20.75$ and $21 \mathrm{eV}$ and the corresponding $E_{c u t}^{26}$ for iron nuclei. For each combination of the injected particle type and $E_{c u t}$ nine values of the spectral index $(\alpha)$, going from 2 to 2.8 in steps of 0.1 , were considered.

Using the upper limit on the integral flux of gamma-rays $\left(I_{\gamma}^{U L}\left(>E_{\gamma}^{t h}\right)\right)$ obtained by H.E.S.S. the upper limit on the cosmic-ray luminosity $L_{C R}^{U L}$ is calculated, by mean of equation (2.8), for each simulated scenario. The upper limit UHECR flux obtained for each source by using equation (2.6) is therefore determined by the injection spectrum shape ( $\alpha$ and $E_{c u t}$ ) and by the calculated $L_{C R}^{U L}$.

The upper limit on the cosmic-ray luminosity of a given point source calculated by using this method is useful only if the contribution of the corresponding UHECR flux to the total flux observed by a given cosmic-ray observatory, which includes all sources in its field of view, is smaller than the upper limit on the UHECR total flux measured by this observatory and calculated at the same CL. Because a given point source contributes in a different way to the total UHECR flux observed by different cosmic-ray observatories, this condition has to be fulfilled considering the observations done by every cosmic-ray observatory relevant in this energy range.

The contribution to the spectrum measured by the Pierre Auger Observatory of the upper limit UHECR flux calculated for NGC 1068, for all scenarios considered, does not fulfill the condition of being lower than the upper limit on the UHECR total flux measured by the Pierre Auger Observatory. This indicates that the upper limit measured by H.E.S.S. is not restrictive enough in order to be used by this method. In this sense, the spectrum measured by cosmic-ray experiments can be used as a classification of the restriction power of the GeV-TeV gamma-ray measurements. For the cases of NGC 7469 and Pictor A this condition is fulfilled for all proton scenarios considered. These sources are analyzed in detail in the next subsections.

Note that Pictor A is only seen by the Pierre Auger Observatory. However NGC 7469 and NGC 1068 are also seen by Telescope Array which is located in the northern hemisphere. In particular, NGC 7469 has a positive declination and is better seen by Telescope Array than by Auger. However, for these two sources it can be seen that the restriction power of 
the data used to estimate the surface detector spectrum of Telescope Array [26] is smaller than the one corresponding to the Pierre Auger Observatory, i.e. the upper limit on the total flux obtained from the surface detector data of Telescope Array is larger than the one corresponding to Auger. This is due to essentially two reasons, the flux observed by Telescope Array is larger than the one observed by Auger and the statistics of Auger is larger than the one corresponding to Telescope Array. Therefore, for the analyses of NGC 7469 and Pictor A only the Auger spectrum is considered.

\subsection{Pictor A}

Pictor A is an interesting Fanarof-Riley II broad emission line radio galaxy [27, 28]. Due to its relative proximity $(z=0.0342)$ it has been extensively observed in many wavelength ranges. Radio observations have shown a relativistic jet with rich hot spots in the termination shock region [17]. Recently, Fermi-LAT has detected GeV gamma-rays $(0.2<\mathrm{E}<300 \mathrm{GeV})$ with a very low flux $\left(F_{0.2<E<300 \mathrm{GeV}}=(5.8 \pm 0.7) \times 10^{-9} \mathrm{~cm}^{-2} \mathrm{~s}^{-1}\right)$ coming from this source [29].

The abundance of data from this source (see Ref. [30] for a compilation) and the possibility of resolving its inner structures make it an important object with which to probe to many of the unsolved questions regarding gamma-ray emission. The broad band emission of Pictor A suggests a synchrotron self-Compton mechanism operating in the source in which $\mathrm{TeV}$ gamma-rays can be produced via inverse-Compton scattering. However the predicted flux is well below the measurements of Fermi-LAT and H.E.S.S. [29].

Regarding the acceleration of the highest energetic particles, Pictor A is also a plausible candidate. A magnetic field of the order of $1 \mu$ Gauss has been measured in filaments with kpc size [31] which guarantees the possibility of accelerating particles up to $E \sim 10^{20} \mathrm{eV}$ according to the Hillas criteria [32]. At the same time, the measured luminosity of the jet $L_{j e t} \sim 10^{43.1} \mathrm{erg} \mathrm{s}^{-1}$ also satisfies the Lovelace [33] condition for acceleration in the jet.

The upper limit on the integral gamma-ray flux of Pictor A obtained by H.E.S.S., at $99.9 \% \mathrm{CL}$, is $I_{\gamma}^{U L}\left(E>E_{\gamma}^{t h}=320 \mathrm{GeV}\right)=2.45 \times 10^{-12} \mathrm{~cm}^{-2} \mathrm{~s}^{-1}$. As explained above, by using this upper limit and equation (2.8), the upper limit on the cosmic-ray luminosity of Pictor A is calculated for all scenarios considered. Then, the upper limit on the UHECR flux is calculated by using the upper limit on the cosmic-ray luminosity.

Figure 1 shows the contribution of the upper limit on the UHECR flux of Pictor A to the total flux observed by the Pierre Auger Observatory. Note that the calculated flux is weighted by the exposure of the Pierre Auger Observatory in the direction of Pictor A (see appendix B for details). The plots on the left column show the results for the cases of only primary protons and on the right column the ones for the cases of only iron nuclei. Each row in figure 1 shows a different cutoff energy of the cosmic rays in the source, $\log \left(E_{\text {cut }}^{p r} / \mathrm{eV}\right)=20,20.5$ and 21 for protons and $\log \left(E_{\text {cut }}^{26} / \mathrm{eV}\right) \cong 21.41,21.91$ and 22.41 for iron nuclei $\left(E_{\text {cut }}^{26}=26 \times E_{p r}\right)$. All plots show the combined cosmic-ray spectrum measured by the Pierre Auger Observatory [34] and the upper limits on the total flux (arrows) at 99.9\% CL obtained from the surface detector data only (number of events in each energy bin and the exposure taken from Ref. [34]), assuming Poisson statistics.

From figure 1 (right column) it can be seen that for several combinations of cutoff energy and spectral index and in many energy bins, the contribution of the upper limit UHECR flux of Pictor A to the total flux observed by Auger, for the case of iron nuclei primaries, is above the upper limit on the total flux of Auger (obtained from the surface detector data). Therefore, the upper limit on the integral flux of gamma-rays measured by H.E.S.S. does 
not impose a valid upper limit on the cosmic-ray luminosity of Pictor A for the case in which iron nuclei are considered for the analysis.

On the other hand, the upper limit measured by H.E.S.S. offers a valid constrain if UHECR protons are injected by the source. From figure 1 (left column) it can be seen that for all scenarios considered regarding proton primaries, the contribution of the upper limit UHECR flux of Pictor A to the total flux observed by Auger is smaller than the upper limit on the total flux of Auger (obtained from the surface detector data). In this case, the upper limit on the integral flux of the gamma-rays determines a valid upper limit on the proton luminosity of Pictor A. Figure 2 shows the upper limit on the proton luminosity of Pictor A as a function of the spectral index for $\log \left(E_{\text {cut }} / \mathrm{eV}\right)=20,20.25,20.5,20.75$, and 21 .

Pictor A has been observed by Fermi-LAT [29] in the energy range from 0.2 to $300 \mathrm{GeV}$. However the upper limit on the integral gamma-ray flux obtained by H.E.S.S. is the most restrictive condition for the proton luminosity of Pictor A. The flux measured by Fermi-LAT in the energy range from 0.2 to $300 \mathrm{GeV}$ is more than one order of magnitude larger than the one derived from the upper limit measured by H.E.S.S., independent of spectral index and cutoff energy.

As mentioned before, upper limits on the photon fraction at the highest energies have been obtained by the past and current cosmic-ray observatories. The most stringent ones come from the observations done by the Pierre Auger Observatory [35-37]. The upper limits on the integral gamma-ray flux obtained by the Pierre Auger Observatory are more than two orders of magnitude larger than the integral gamma-ray flux calculated from the upper limit obtained by H.E.S.S., in the same energy range, independent of spectral index and cutoff energy. Therefore, also in this case the gamma-ray observations at $\mathrm{GeV}-\mathrm{TeV}$ energies provide the most restrictive upper limit on the proton luminosity of the source.

\section{$3.2 \quad$ NGC 7469}

NGC 7469 is a well known spiral galaxy [38] with a Seyfert 1 nucleus embedded in a ring of starburst activity. At radio frequencies, its structure shows an unresolved central component surrounded by a ring of star forming regions [39].

The upper limit on the integral gamma-ray flux of NGC 7469 obtained by H.E.S.S. at $99.9 \% \mathrm{CL}$ is $I_{\gamma}^{U L}\left(E>E_{\gamma}^{t h}=330 \mathrm{GeV}\right)=1.38 \times 10^{-12} \mathrm{~cm}^{-2} \mathrm{~s}^{-1}$. Also for this source, the upper limit obtained by H.E.S.S. offers a valid constraint if UHECR protons are emitted by the source. As for the case of Pictor A, if iron nuclei are considered the contribution of the upper limit UHECR flux of NGC 7469 to the total flux measured by Auger is larger than the upper limit UHECR flux of Auger obtained from the surface detector data. Figures 3 shows the upper limits on the proton luminosity as a function of the spectral index for the same values of the cutoff energies as the ones considered for Pictor A.

Also for NGC 7469 the upper limits on the integral gamma-ray flux obtained by the Pierre Auger Observatory, at the highest energies, are more than two orders of magnitude larger than the integral gamma-ray flux calculated from the upper limit obtained by H.E.S.S., in the same energy range, independent of spectral index and cutoff energy. Therefore, also for this source the gamma-ray observations at $\mathrm{GeV}-\mathrm{TeV}$ energies provide the most restrictive upper limit on its proton luminosity. 


\section{Conclusions}

In this work a new method to infer an upper limit on the cosmic-ray luminosity of individual sources from $\mathrm{GeV}-\mathrm{TeV}$ gamma-ray observations has been presented. This new method is based on the fact that the UHECR produce gamma-rays when they propagate from the sources on their way to Earth. The gamma-rays generated in this manner contribute to the total flux observed at Earth.

Measurements done by ground and space based experiments impose upper limit on the integral flux of GeV-TeV gamma-rays of some sources. Using a conservative approach of no intrinsic gamma-ray emission of the source it is possible to calculate an upper limit on the cosmic-ray luminosity of individual sources.

We have studied in detail the cases of Pictor A and NGC 7469. We have calculated an upper limit on the proton luminosity of the two sources as a function of the index of the injected spectrum for several values of the cutoff energies (figures 2 and 3). We have also shown that the $\mathrm{GeV}-\mathrm{TeV}$ gamma-ray upper limits obtained by H.E.S.S. impose the most restrictive condition on the proton luminosity of the sources.

The results presented in this work illustrate one caveat of the multi-messenger techniques to study the origin of cosmic rays at the highest energies and gamma-rays at $\mathrm{GeV}-\mathrm{TeV}$ energies. The method elaborated here has its motivation increased by the future Cherenkov Telescope Array which will allow the observation of a much larger number of extragalactic objects than the ones observed at present with a much lower sensitivity.

\section{A Simulations}

In this work the propagation of cosmic-ray particles from the source to Earth is simulated with CRPropa [24]. CRPropa is one of the most complete public software packages to model the propagation of nuclei in the intergalactic media considering the most relevant particle interactions and radiation backgrounds.

CRPropa also propagates the secondary photons generated by the propagation of the nuclei, which is the main interest of this paper. The most important effect is the pair production due to the interaction of high energy nuclei with the photon backgrounds. This effect is implemented as a continuous energy loss according to the parametrization given in Refs. [911]. Other important effects which produces $\mathrm{GeV}-\mathrm{TeV}$ gamma rays are also considered in CRPropa, like, photo-pion production and the subsequent $\pi^{0}$ decay.

The injection spectrum assumed in this work consists of a power law with an exponential cutoff (see equation (2.1)). We have modified CRPropa in order to include the exponential cutoff.

The propagation of the particles is done with the one dimensional option in CRPropa. The SOPHIA [40] interaction package, available in CRPropa, is used allowing a detailed simulation of the photo-pion interaction process. Also, for each studied case $10^{7}$ particles are injected at the source position and propagated to the Earth.

Standard cosmology is assumed for the calculations, the Hubble parameter is given by $H(z)=H_{0} \sqrt{\Omega_{m}(1+z)^{3}+\Omega_{\Lambda}}$ with $H_{0}=72 \mathrm{~km} \mathrm{~s}^{-1} \mathrm{Mpc}^{-1}, \Omega_{m}=0.26$, and $\Omega_{\Lambda}=0.74$.

In the present calculations it is assumed that the Intergalactic Magnetic Field (IGMF) is negligible. In principle, the presence of a non-zero IGMF modifies the electromagnetic cascades mainly due to the deflection of the electrons and positrons in this field. These effects are negligible if the magnitude of the IGMF is $\lesssim 10^{-14} \mathrm{G}$ [8]. Recently, a lower limit 
of order of $10^{-15} \mathrm{G}$ has been obtained from gamma-ray observations of distant blazars [4143]. However, in Ref. [44] it has been suggested that a null IGMF cannot be excluded due to systematic effects.

Figure 4 illustrates the simulations done with CRPropa. The distance of Pictor A is used in this example. The spectral index is $\alpha=2.3$ and two different pure compositions injected by the source are considered, protons and iron nuclei with cutoff energies $E_{c u t}^{p}=10^{20.5} \mathrm{eV}$ and $E_{c u t}^{26}=26 \times 10^{20.5} \mathrm{eV}$, respectively. The luminosity of the source is taken as $L_{C R}=2.1 \times 10^{44}$ $\mathrm{erg} \mathrm{s}^{-1}$. The figure shows the flux at Earth corresponding to cosmic rays and gamma-rays for the proton and iron nuclei cases. The propagated spectra shown in this figure are calculated from $K$ and $P$ used in section 2.

\section{B Contribution of a point source to the measured UHECR flux}

Cosmic ray observatories detect particles coming from all directions in the sky over large periods of time. Therefore, each individual cosmic-ray source contributes in a different way to the observed spectrum. The flux of a given source is weighted by a factor that depends on its position in the sky, on the location of the observatory, and on the specific characteristics of the detectors and methods used for the data analysis.

The differential number of detected cosmic-ray particles that come from one given source is given by

$$
\frac{d N_{s}}{d E}=\Phi_{s}(E) A \varepsilon_{0} \int_{0}^{T_{o b s}} d t \Theta\left(\theta_{\max }-\theta_{s}(t)\right) \cos \theta_{s}(t),
$$

where subscript $s$ is used to specify a given source, $\Phi_{s}(E)$ is the source flux at Earth, $A$ is the area of the observatory, $T_{o b s}$ is the observation time, $\theta_{s}$ is the source zenith angle, and $\varepsilon_{0}$ is the efficiency of the observatory. Usually $\varepsilon_{0}$ depends on primary energy and zenith angle, however $\varepsilon_{0}$ is taken as a constant which is true for the Pierre Auger Observatory for primary energies $E \gtrsim 10^{18.5} \mathrm{eV}$ and zenith angles $\theta \lesssim 60^{\circ}$ [45] and also for the Telescope Array for $E \gtrsim 10^{18.8} \mathrm{eV}$ and $\theta \lesssim 45^{\circ}[26]$. Here $\Theta(x)$ is the Heaviside function $(\Theta(x)=1$ for $x>0$ and $\Theta(x)=0$ otherwise) and it is assumed that the events used to calculate the measured spectrum are such that $\theta \leq \theta_{\max }$.

The observed cosmic-ray flux $\left(J_{C R}^{s}(E)\right)$ is obtained dividing the differential number of events by the exposure $(\mathcal{E})$

$$
J_{C R}^{s}(E)=\frac{1}{\mathcal{E}} \frac{d N_{s}}{d E}
$$

and for a constant efficiency the exposure can be written as

$$
\mathcal{E}(E)=T_{\text {obs }} A \varepsilon_{0} \pi \sin ^{2} \theta_{\text {max }} \Theta\left(E-E_{0}\right),
$$

where $E_{0}$ is the threshold energy used to calculate the energy spectrum.

Combining equations (B.1), (B.2), and (B.3) the contribution of a point source to the total flux can be written as

$$
J_{C R}^{s}(E)=W_{s} \Phi_{s}(E)
$$

where $W_{s}=\omega_{s} /\left(\pi \sin ^{2} \theta_{\max }\right)$ and

$$
\omega_{s}=\frac{1}{T_{o b s}} \int_{0}^{T_{o b s}} d t \Theta\left(\theta_{\max }-\theta_{s}(t)\right) \cos \theta_{s}(t)
$$

For an analytical expression of $\omega_{s}$ see Ref. [46]. 


\section{Acknowledgments}

VdS thank the support of the Brazilian population via CNPq and FAPESP (2010/195146). VdS is also in debt with Edivaldo Moura Santos and Rita de Cássia dos Anjos. ADS is member of the Carrera del Investigador Científico of CONICET, Argentina. The work of ADS is supported by CONICET PIP 114-201101-00360 and ANPCyT PICT-2011-2223, Argentina. The authors thank the Pierre Auger Collaboration for permission to use their data prior to journal publication.

\section{References}

[1] The Pierre Auger Collaboration, Update on the correlation of the highest energy cosmic rays with nearby extragalactic matter, Astropart. Phys., 34 (2010) 314-326.

[2] Fukushima, M., Measurement of Ultra-High Energy Cosmic Rays: An Experimental Summary and Prospects, Proceedings of the symposium UHECR-2012 at CERN, arXiv:1302.5893.

[3] The Fermi-LAT Collaboration, Fermi Large Area Telescope Second Source Catalog, Astrophys. J. Suppl., 199 (2012) 31.

[4] Gabici, S. and Aharonian, F., Pointlike Gamma Ray Sources as Signatures of Distant Accelerators of Ultrahigh Energy Cosmic Rays, Phys. Rev. Lett., 95 (2005) 251102.

[5] Armengaud, E., Sigl, G., and Miniati, F., Gamma Ray Astronomy with Magnetized Zevatrons, arXiv:astro-ph/0511277.

[6] Ferrignoa, C., Blasi, P., and De Marco, D., High energy gamma ray counterparts of astrophysical sources of ultra-high energy cosmic rays, Astropart. Phys., 23 (2005) 211-226.

[7] Kotera, K., Allard, D., and Lemoine, M., Detectability of ultrahigh energy cosmic-ray signatures in gamma-rays, Astronomy and Astrophysics, 527 (2011) A54.

[8] Ahlers, M. and Salvado, J., Cosmogenic gamma rays and the composition of cosmic rays, Phys. Rev. D, 84 (2011) 085019.

[9] Puget, J., Stecker, F., and Bredekamp, J., Photonuclear Interactions of Ultrahigh-Energy Cosmic Rays and their Astrophysical Consequences, Astrophys. J., 205 (1976) 638.

[10] Blumenthal, G., Energy loss of high-energy cosmic rays in pair-producing collisions with ambient photons, Phys. Rev. D, 1 (1970) 1596.

[11] Kelner, S., Aharonian, F., Energy spectra of gamma rays, electrons, and neutrinos produced at interactions of relativistic protons with low energy radiation, Physical Review D, 78 (2008) 034013.

[12] De Angelis, A., Galanti, G., and Roncadelli, M., Transparency of the Universe to gamma rays, Monthly Notices of the Royal Astronomical Society, 432 (2013) 3245.

[13] Essey, W., Kalashev, O., Kusenko, A., and Beacom, J., Secondary Photons and Neutrinos from Cosmic Rays Produced by Distant Blazars, Phys. Rev. Lett., 104 (2010) 141102.

[14] Essey, W. and Kusenko, A., A new interpretation of the gamma-ray observations of distant active galactic nuclei, Astropart. Phys., 33 (2010) 81-85.

[15] Essey, W., Kalashev, O., Kusenko, A., and Beacom, J., Role of Line-of-sight Cosmic-ray Interactions in Forming the Spectra of Distant Blazars in TeV Gamma Rays and High-energy Neutrinos, Astrophys. J., 731 (2011) 51.

[16] H.E.S.S. Collaboration, Observations of the Crab nebula with HESS, Astronomy and Astrophysics, 457 (2006) 899. 
[17] Wilson, A. S., Young, A. J., and Shopbell, P. L., Chandra X-Ray Observations of Pictor A: High-Energy Cosmic Rays in a Radio Galaxy, ApJ, 547 (2001) 740.

[18] CTA Consortium,Design Concepts for the Cherenkov Telescope Array, Experimental Astronomy, 32 (2011) 193-316.

[19] Takahashi, Y. and the JEM-EUSO Collaboration, The JEM-EUSO mision, New Journal of Physics, 11 (2009) 065009.

[20] The Pierre Auger Collaboration, The Next Frontier in UHECR Research with an Upgraded Pierre Auger Observatory, arXiv:1307.0226.

[21] Becker, J., High-energy neutrinos in the context of multimessenger physics, Physics Report $\mathbf{4 5 8}$ (2008) 173.

[22] Allard, D., Extragalactic propagation of ultrahigh energy cosmic-rays, Astropart. Phys., 39-40 (2012) 33 .

[23] Kachelriess, M., Ostapchenko, S., Tomas, R., Multi-messenger astronomy with Centaurus A, Int. J. Mod. Phys. D, 18 (2009) 1591.

[24] Kampert, K., et al., CRPropa 2.0 - a Public Framework for Propagating High Energy Nuclei, Secondary Gamma Rays and Neutrinos, Astopart. Phys., 42 (2013) 41.

[25] Aharonian, F. et al, Upper limits from HESS active galactic nuclei observations in 2005-2007, Astronomy and Astrophysics, 478 (2008) 387.

[26] Ivanov, D., Energy spectrum measured by the Telescope Array Surface Detector, PhD thesis 2012, available at http://www.telescopearray.org/index.php/research/publications/theses.

[27] Eracleous, M. and Halpern, J., Accurate Redshifts and Classifications for 110 Radio-Loud Active Galactic Nuclei, The Astrophysical Journal Supplement Series, 150 (2004) 181.

[28] Liu, F. and Zhang, Y., A new list of extra-galactic radio jets,Astronomy\& Astrophysics, 381 (2002) 757.

[29] Anthony, B. and Adams, J., Discovery of gamma-ray emission from the Broad Line Radio Galaxy Pictor A, Monthly Notices of the Royal Astronomical Society, 421 (2012) 2303.

[30] Tingay, S., Lenc, E., Brunetti, G., and Bondi, M., A high resolution view of the jet termination shock in a hot spot of the nearby radio galaxy Pictor $A$ : implications for $X$-ray models of radio galaxy hot spots, The Astronomical Journal, 136 (2008) 2473.

[31] Perley, R., Röser, H., and Meisenheimer, K., The radio galaxy Pictor A - a study with the VLA, Astron. Astrophys., 328 (1997) 12.

[32] Hillas, A. M. Cosmic Rays: Recent Progress and some Current Questions, (2006) astro-ph:0607109.

[33] Lovelace, R. V. E., Dynamo model of double radio sources, Nature, 262 (1976) 649.

[34] Salamida, F. for The Pierre Auger Collaboration, Update on the measurement of the CR energy spectrum above $10^{18}$ eV made using the Pierre Auger Observatory, Proceedings of the 32nd International Cosmic Ray Conference, Beijing, China, 2011.

[35] The Pierre Auger Collaboration, Upper limit on the cosmic-ray photon flux above $10^{19}$ eV using the surface detector of the Pierre Auger Observatory, Astropart. Phys., 29 (2008) 243.

[36] Settimo, M. for The Pierre Auger Collaboration, An update on a search for ultra-high energy photons using the Pierre Auger Observatory, Proceedings of 32nd International Cosmic Ray Conference, Beijing, China, 2011, arXiv:1107.4805.

[37] The Pierre Auger Collaboration, An upper limit to the photon fraction in cosmic rays above $10^{19}$ eV from the Pierre Auger Observatory, Astropart. Phys., 27 (2007) 155. 
[38] Anderson, K., Spectrophotometry of Eight Seyfert Galaxies, Astrophysical Journal, 162 (1970) 743.

[39] Wilson, A., Helfer, T., Haniff, H., and Ward, M., The starburst ring around the Seyfert nucleus in NGC 7469, Astrophysical Journal, 381 (1991) 79.

[40] Mücke, A., Engel, R., Rachen, J., Protheroe, R., and Stanev, T., Monte Carlo simulations of photohadronic processes in astrophysics, Comp. Phys. Commun., 124 (2001) 290.

[41] Taylor, A., Vovk, I., and Neronov, A., EGMF Constraints from Simultaneous GeV-TeV Observations of Blazars, Astronomy and Astrophysics, 529 (2011) A114.

[42] Neronov, A. and Vovk, I., Evidence for strong extragalactic magnetic fields from Fermi observations of TeV blazars, Science, $\mathbf{3 2 8}$ (2010) 73.

[43] Tavecchio, F., et al., Extreme TeV blazars and the intergalactic magnetic field, Monthly Notices of the Royal Astronomical Society, 414 (2011) 3566.

[44] Arlen, T., et al., Intergalactic Magnetic Fields and Gamma Ray Observations of Extreme TeV Blazars, arXiv:1210.2802.

[45] The Pierre Auger Collaboration, Trigger and aperture of the surface detector array of the Pierre Auger Observatory, Nuclear Instruments and Methods in Physics Research A, 13 (2010) 29.

[46] Sommers, P., Cosmic ray anisotropy analysis with a full-sky observatory, Astropart. Phys., 14 (2001) 271. 


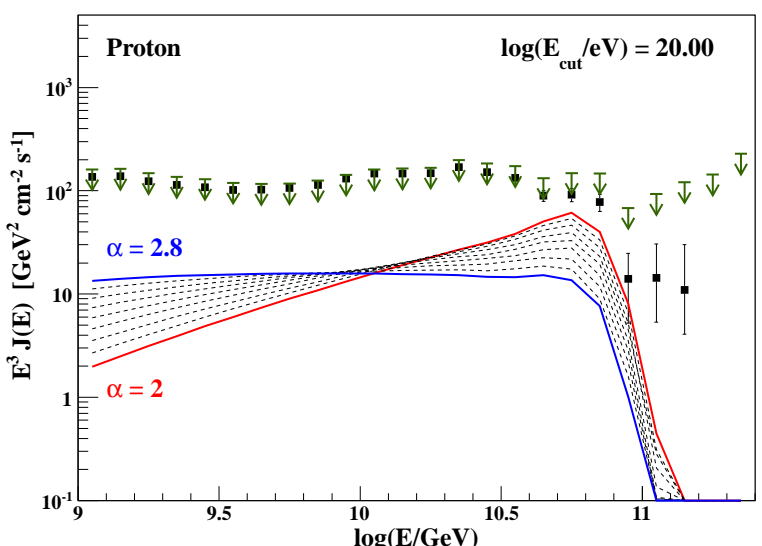

(a) $\operatorname{Pr}-E_{\text {cut }}=10^{20} \mathrm{eV}$

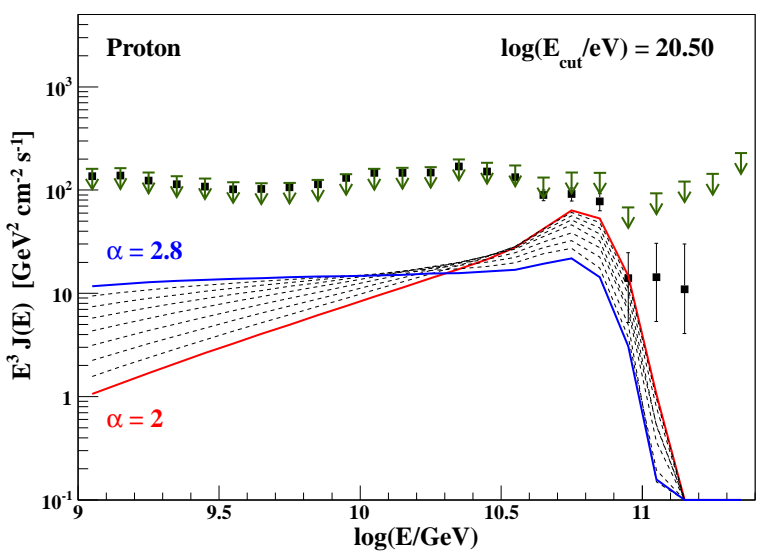

(c) $\operatorname{Pr}-E_{\text {cut }}=10^{20.5} \mathrm{eV}$

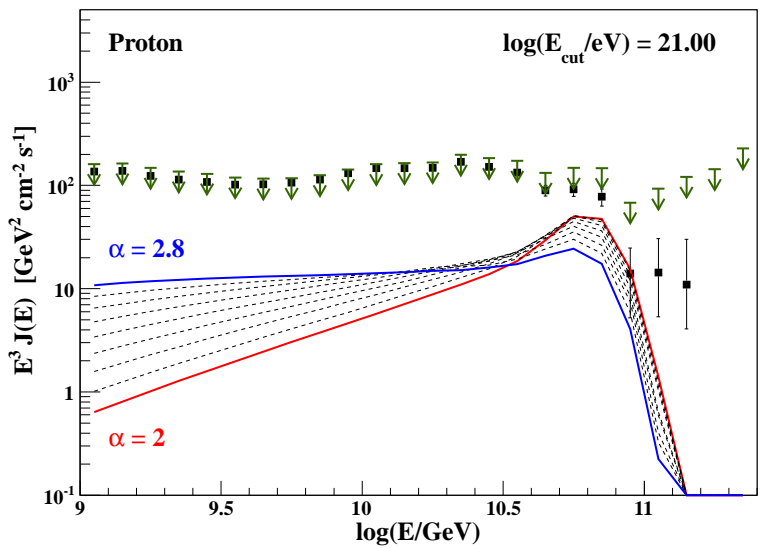

(e) $\operatorname{Pr}-E_{\text {cut }}=10^{21} \mathrm{eV}$

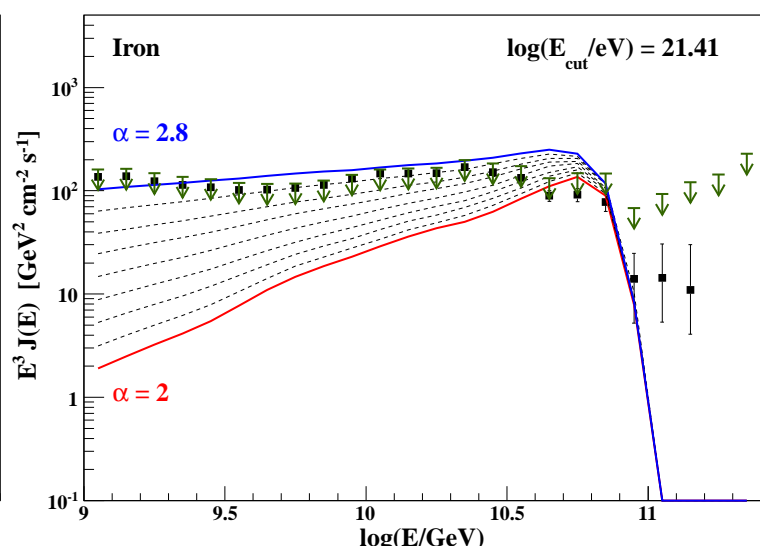

(b) $\mathrm{Fe}-E_{\text {cut }}=26 \times 10^{20} \mathrm{eV}$

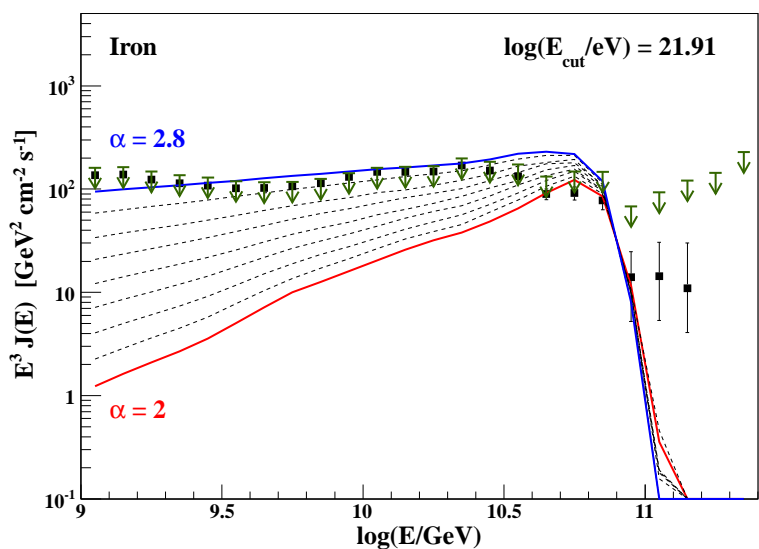

(d) $\mathrm{Fe}-E_{\text {cut }}=26 \times 10^{20.5} \mathrm{eV}$

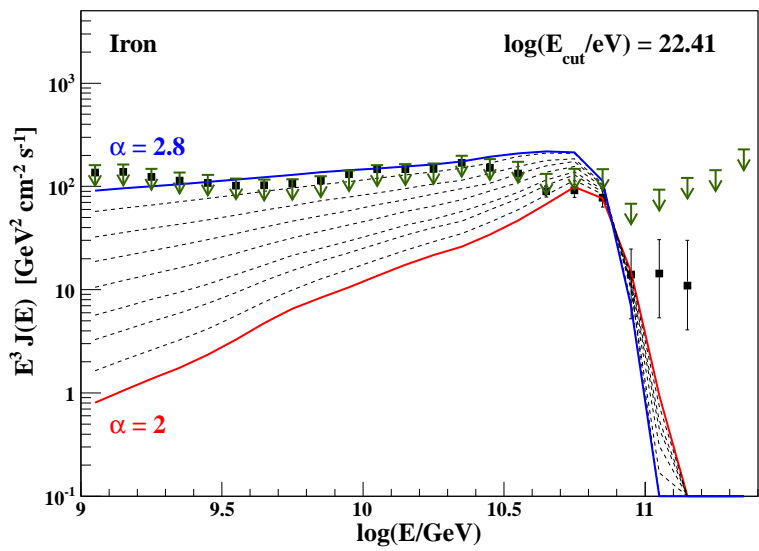

(f) $\mathrm{Fe}-E_{\text {cut }}=26 \times 10^{21} \mathrm{eV}$

Figure 1: Upper limit on the UHECR cosmic ray flux for Pictor A calculated by using the upper limit on the integral flux of $\mathrm{GeV}-\mathrm{TeV}$ gamma-rays measured by H.E.S.S.. The points with error bars correspond to the combined spectrum measured by the Pierre Auger Observatory and the arrows correspond to the upper limit on the flux, at 99.9\% CL, obtained from the surface detector data, assuming Poisson statistics. Left column: Figures (a), (c), and (e) correspond to proton primaries. Right column: Figures (b), (d), and (f) correspond to iron primaries. 


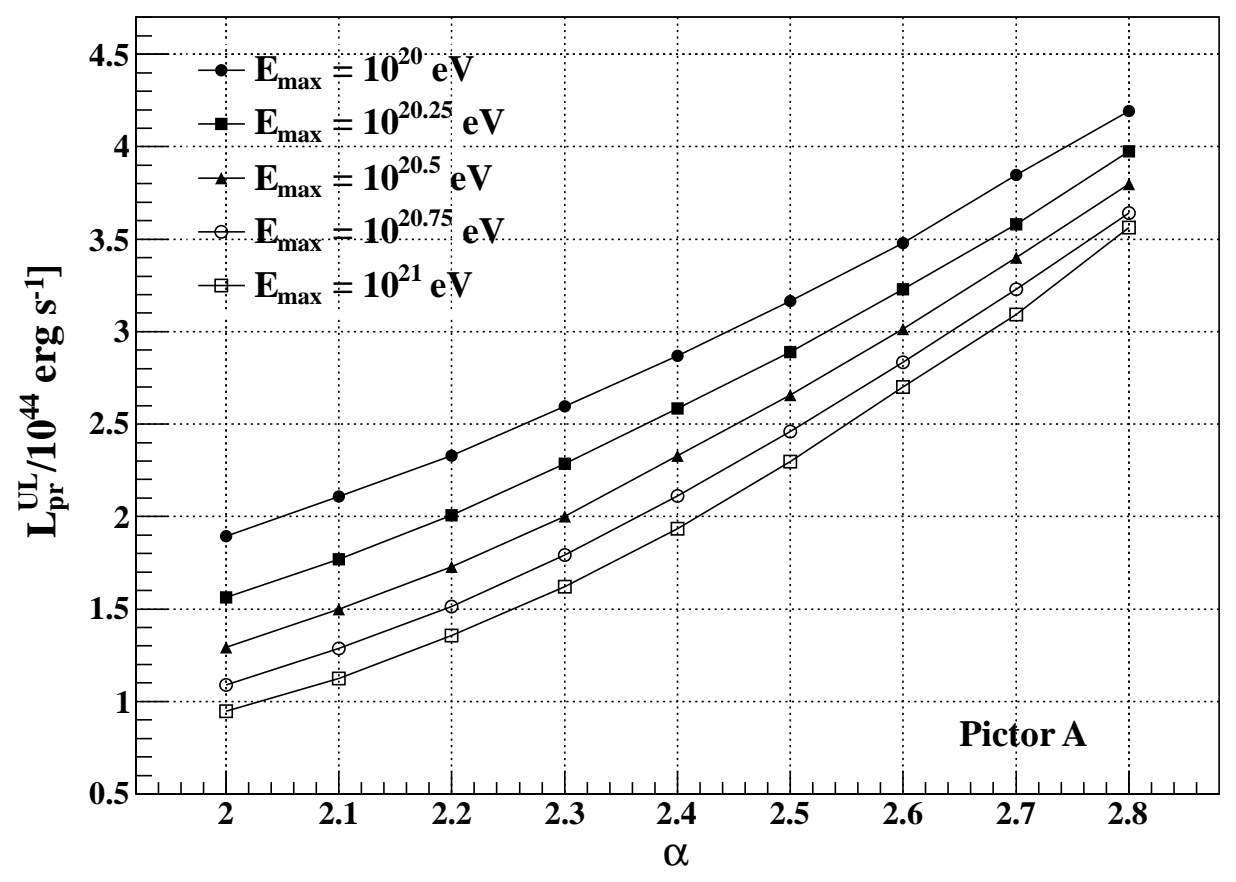

Figure 2: Upper limit on the proton luminosity of Pictor A as a function of the spectral index of the injection spectrum. The calculation is based on the measured upper limit on the integral flux of GeV-TeV gamma-rays obtained by H.E.S.S.. The cutoff energies are: $\log \left(E_{\text {cut }} / \mathrm{eV}\right)=20,20.25,20.5,20.75$, and 21 . 


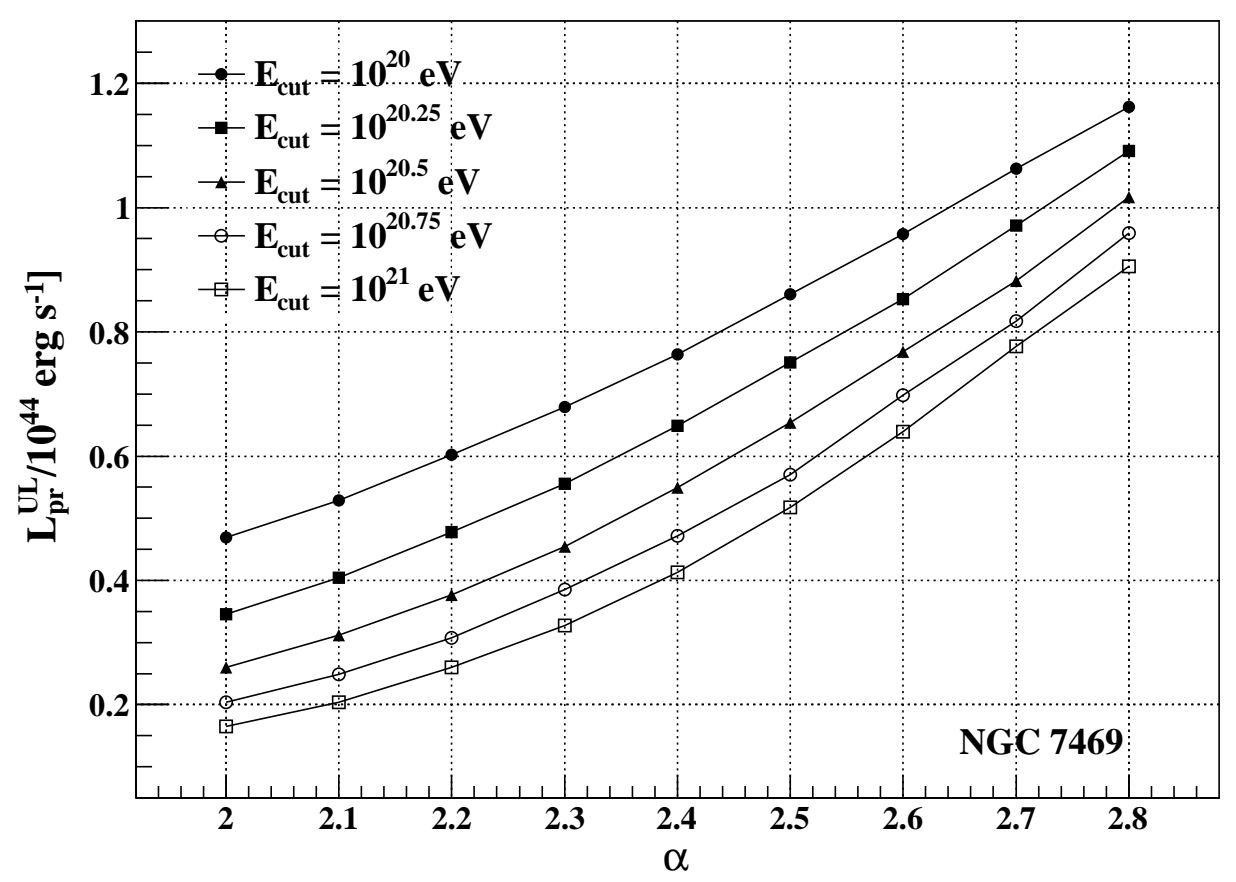

Figure 3: Upper limit on the proton luminosity of NGC 7469 as a function of the spectral index of the injection spectrum. The calculation is based on the measured upper limit on the integral flux of GeV-TeV gamma-rays obtained by H.E.S.S.. The cutoff energies are: $\log \left(E_{\text {cut }} / \mathrm{eV}\right)=20,20.25,20.5,20.75$, and 21 .

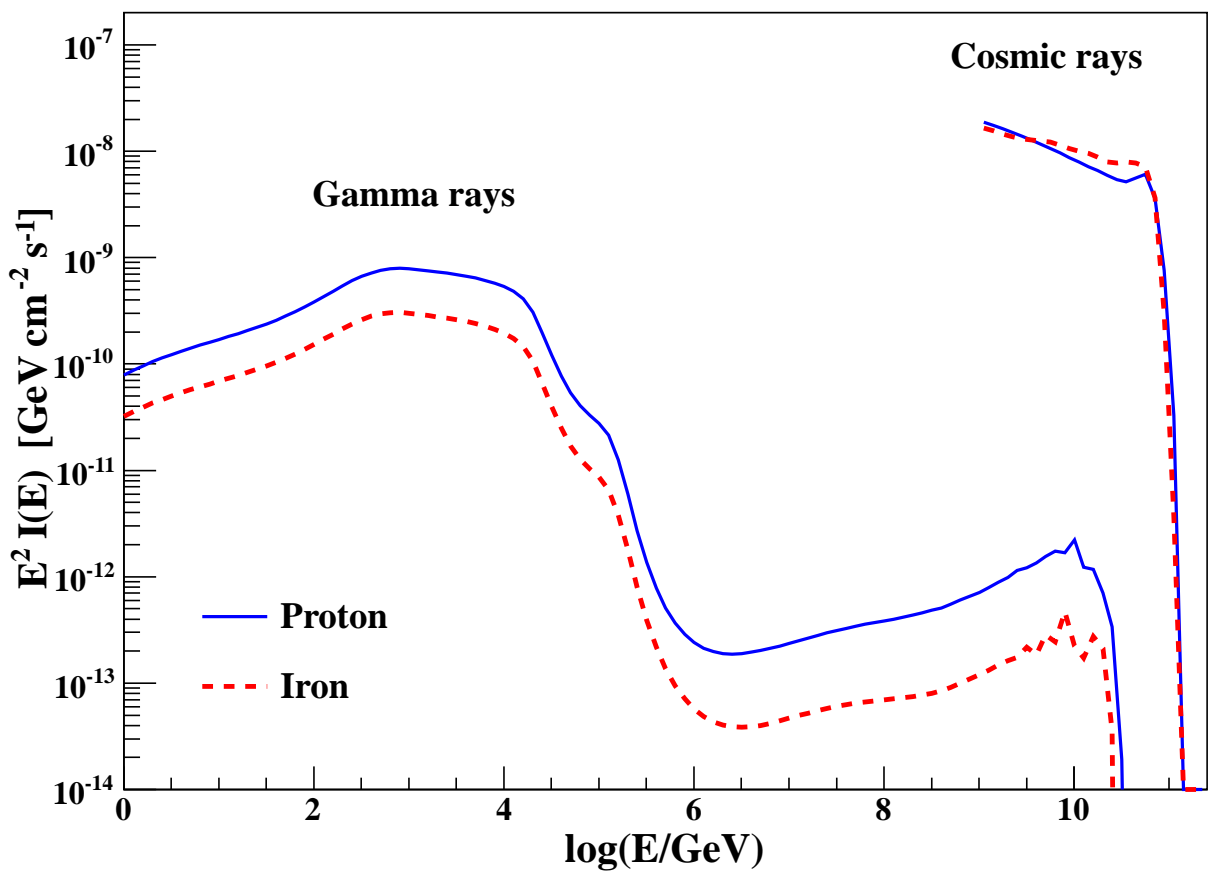

Figure 4: Cosmic-ray and gamma-ray fluxes as a function of the logarithm of the energy for a source at the distance of Pictor A obtained with CRPropa. Solid lines correspond to an injection spectra composed by proton and dashed lines to iron nuclei. 